\title{
DESIGNING A CONSTRUCTIVE GUIDANCE SYSTEM WITH REAL TIME OBJECT DETECTION FOR VISUALLY IMPAIRED
}

\author{
Mrs.N.Deepika \\ Assistant Professor, Department of Computer Science and Engineering, DMI College of \\ Engineering \\ Ms Joypriyanka.M \\ Department of Computer Science and Engineering, DMI College of Engineering,
}

Article DOI: https://doi.org/10.36713/epra6178

\begin{abstract}
This paper proposes an effective device for visually impaired person in order to have betterment in their routine life. The spread of visually impairment is very sensitive issue worldwide. Here we develop a Smart system for visually impaired, that make use of ultrasonic sensor and camera. The most point of this work is to plan a voicebased cautioning framework for the outwardly impeded individuals. Visually impaired individual's finds navigation difficult as they struggle every day in performing actions for bypassing obstacles and hurdles in their regular life. In order to help visually impaired people navigate safely and quickly this system is proposed. Ultrasonic sensor is set on the exhibition which is utilized for impediment location with separate sign. The camera is set to detect the object before the outwardly impeded individuals and alarm them utilizing APR voice module. This system prevents the visually impaired people accidents and identifies the object in front of them. The scope of the work is extracting the object's image and processing them in the knowledge base and interpret the captured one. Assistive Innovation for individuals with outwardly disabilities is anticipated to develop at a quick pace and affect the lives of people having visual impedance and the elderly ways previously is not possible.
\end{abstract}

KEY WORDS: SURF-Speedup Robust Feature, VPN-Visual Handling Unit, NCS-Neural Compute Adhere, APR-Apache Portable Runtime.

\section{I.INTRODUCTION}

Visual impairment and visual impairment may be a worldwide issue with imperative socio-economic results that have demonstrated impacts on the quality of life of people, and more often than not force extraordinary family-related and socio-economic losses [1]. Blind people make maximum use of sound not only to know the obstacle presence, but also how dangerous it is, in order to avoid it effectively, the devices use acoustical sounds in arrange to speak to the visual data recognized by the sensors and manufactured vision frameworks[2]. The objective of this paper is to create a low-cost shrewdly framework for directing people who are dazzle or somewhat located by giving data around the natural situation of static and dynamic objects around them [3]. The most capacities of this framework are way sign and environment acknowledgment. However, many obstacle detection and avoidance systems have been introduced during the last decade to assist visually impaired to navigate in known or unknown, indoor and outdoor environments [4-7]. This route can basically be categorized as vision improvement, vision substitution and vision substitution. Vision replacement systems provide the visual cortex of the human brain with the information either directly or via the optic nerve. Vision substitution and Vision upgrade frameworks have nearly same working 


\section{EPRA International Journal of Research and Development (IJRD) \\ Volume: 6 | Issue: 1 | January 2021 \\ - Peer Reviewed Journal}

standards with regard to environment location handle; in any case, each gives the natural data differently. [8-12]Vision improvement puts the data in a visual way, though vision substitution regularly employments tangible or sound-related recognition or both. We propose a Smart system to help such visually impaired people in their basic activities of life only since one needs to utilize their eyes is not cruel that one needs vision [13-15]. Eyesight is one of the fundamental human faculties, and it plays a critical part in human recognition around the encompassing environment. For visually impaired people to be able to provide, experience their vision, imagination mobility is necessary The Worldwide Classification of Maladies 11 (2018) classifies vision impedance into two bunches, remove and close showing vision impairment. Globally; the leading causes of vision impairment are uncorrected refractive errors, cataract, age-related macular degeneration, glaucoma, diabetic retinopathy, and corneal opacity, trachoma, and eye injuries. It limits outwardly disabled capacity to explore, perform regular errands, and influence their quality of life and capacity to connect with the encompassing world upon unaided. With the headway in advances, assorted arrangements have been presented such, as the Eye- ring extend, the content acknowledgment framework, the hand signal, and confront acknowledgment framework, etc. Be that as it may, these arrangements have impediments such as heavyweight, costly, less vigour, more acknowledgment, etc. consequently, progressed methods must advance to assist them[19,20]. So, we propose a framework built on the breakthrough of picture preparing and machine learning.

\section{II.RELATED WORK}

In later a long time, a few infrared-enabled profundity sensor-based and visual sensor-based frameworks have been created to assist the Visually Impaired in identifying and maintaining a strategic distance from impediments. Visual sensor based frameworks utilize stereo or monocular cameras to procure image data of the encompassing environment and analyse it to estimate obstacle positions. These arrangements are by and large cost effective, available, require small or no foundation, are typically wearable, and can as a rule be introduced or embedded into existing portable computing gadgets. Be that as it may, their performance break down quickly in uncontrolled real-world environments due to imaging variables such as movement blur, image determination, video commotion, etc., as well as changes in conditions such as brightening, introduction and scale. Other limitations incorporate helplessness to impediment issues and high computational fetched. Too, stereo cameras are relatively costly and require exact calibration. [11]

\section{A.WORKING PRINCIPLE}

In our proposed system, we've Arduino UNO microcontroller which acts as brain of our system, hence entire systems program is stored in it. We've two units, 1. Walk stick unit 2. Head Unit. The Walk stick consists camera to capture the image of the thing and send the knowledge to controller via serial communication. Ultrasonic sensor determines the space between ground and stick just in case of staircase they approach, these data transmitted via zigbee to the top Unit. Where the APR module is employed to store voice messages it play through speaker depending upon the info received via zigbee.

\section{BLOCK DIAGRAM}

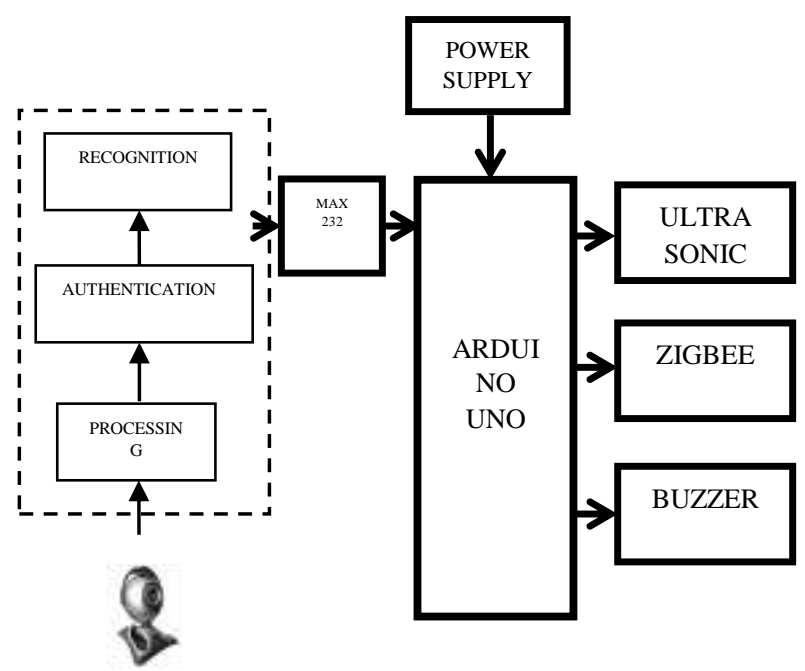

FIG: 1 ARCHITECTURE DIAGRAM

\section{HEAD UNIT}

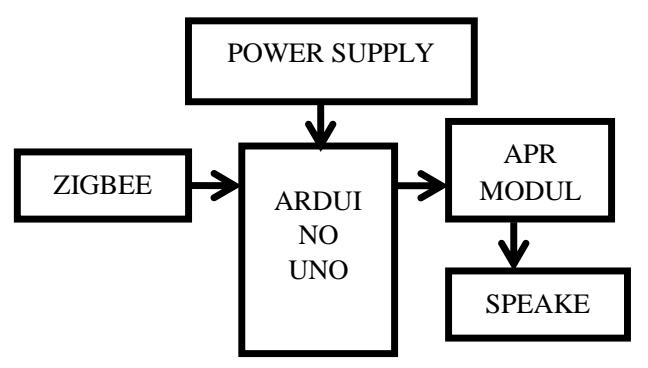

FIG: 2 HEAD UNIT DIAGRAM 


\section{SJIF Impact Factor: 7.001| ISI I.F.Value:1.241| Journal DOI: 10.36713/epra2016 ISSN: 2455-7838(Online) EPRA International Journal of Research and Development (IJRD)

Overall working principle of the walking stick portrays the overall view of the blind stick. The adhere consists of eight ultrasonic sensors in the front side inorder to cover the encompassed region (front, cleared out and right side) of an outwardly impeded individual and the sensors recognized separate of the impediment is send to the client earphone by means of a portable app.

\section{B.HARDWARE}

Arduino is open-source equipment. The equipment reference plans are conveyed beneath an Imaginative Commons Attribution Share-Alike 2.5 permit and are accessible on the Arduino site. Format and generation records for a few forms of the equipment are too accessible [2].

In spite of the fact that the equipment and computer program plans are openly accessible beneath copy left licenses, the engineers have asked the title Arduino to be elite to the official item and not be utilized for inferred works without authorization. The official arrangement archive on utilize of the Arduino title emphasizes that the venture is open to consolidating work by others into the official item. A few Arduino-compatible items commercially discharged have maintained a strategic distance from the extend title by utilizing different names finishing in-duino. An early Arduino board with an RS-232 serial interface (upper cleared out) and an Atmel ATmega8 microcontroller chip (dim, lower right); the 14 computerized I/O pins are at the beat, the 6 analog input pins at the lower right, and the control connector at the lower cleared out.

Most Arduino sheets include of an Atmel 8-bit AVR microcontroller (ATmega8, ATmega168, ATmega328, ATmega1280, ATmega2560) with moving entireties of streak memory, pins, and highlights. The 32-bit Arduino Due, based on the Atmel SAM3X8E was presented in 2012. The sheets utilize single or double-row pins or female headers that encourage associations for programming and joining into other circuits. These may interface with add-on modules named shields. Different and conceivably stacked shields may be exclusively addressable by means of an $\mathrm{I}^{2} \mathrm{C}$ serial transport. Most sheets incorporate a $5 \mathrm{~V}$ direct controller and a 16 $\mathrm{MHz}$ precious stone oscillator or ceramic resonator. A few plans, such as the LilyPad, run at $8 \mathrm{MHz}$ and apportion with the on-board voltage controller due to particular form-factor confinements. [2]

Arduino microcontrollers are pre-programmed with a boot loader that disentangles uploading of programs to the on-chip streak memory. The default boot loader of the Arduino UNO is the optiboot boot loader. Sheets are stacked with program code by means of a serial association to another computer. A few serial Arduino sheets contain a level shifter circuit to change over between RS-232 rationale levels and transistor-transistor rationale (TTL) level signals. Current Arduino sheets are modified by means of Widespread Serial Transport (USB) actualized utilizing USB-to-serial connector chips such as the FTDI FT232. A few sheets, such as latermodel Uno sheets, substitute the FTDI chip with a partitioned AVR chip containing USB-to-serial firmware, which is reprogrammable by means of its claim ICSP header. Other variations, such as the Arduino Scaled down and the informal Boarduino, utilize a separable USB-to-serial connector board or cable, Bluetooth or other strategies. When they are being utilized with standard microcontroller contraptions, rather than the Arduino IDE, standard AVR in-system programming (ISP) programming has been utilized. An official Arduino Uno R2 with portrayals of the I/O areas

The Arduino board uncovered most of the microcontroller's I/O pins for utilize by other circuits. The Diecimila, [a] Duemilanove, [b] and current Uno[c] give 14 computerized I/O pins, six of which can deliver pulse-width balanced signals, and six analog inputs, which can too be utilized as six computerized I/O pins. These pins are on the best of the board, through female 0.1-inch $(2.54 \mathrm{~mm})$ headers. A few plug-in application shields are moreover commercially accessible. The Arduino Nano and Arduino-compatible Revealed Bones Board and Boarduino sheets may grant male header pins on the underside of the board that can plug into patch less breadboards.

Numerous Arduino-compatible and Arduinoderived sheets exist. A few are practically proportionate to an Arduino and can be utilized traded. Numerous upgrades the fundamental Arduino by including yield drivers, regularly for utilize in school-level instruction, to streamline making buggies and little robots. Others are electrically proportionate but alter the frame figure, in some cases holding compatibility with shields, now and then not. A few variations utilize distinctive processors, of shifting compatibility.

\section{III.PROPOSED \& ALGORITHM}

The most advantage of the framework is that it makes a difference the daze individuals in both indoor and open air, care-free navigation. The devices placed within the stick makes it comfortable and straightforward to handle. The smart stick helps in detecting obstacles placed at a distance ahead of the user. The framework is reasonable for both indoor and open air environment. The information regarding obstacles is given through voice alerts, eliminates the difficulty 
RASPBERRY PI Ultra sonic SENSOR Voice synthesizer Battery Micro phone Buzzer Rf receive Camera Water sensor. (9v dc) $\mathrm{RF}$ transmitter Button Worldwide Diary of Immaculate and connected math Uncommon Issue 4534 of understanding vibration designs which was utilized in prior systems. The system may be a moderate budget mobile navigational aid for the visually impaired [2].

$>$ In our proposed system, the blind people has camera enabled walking stick to examine which object it is.

$>$ Here, we have distance sensor detects depth or height the staircase step and tells to people.

\section{E. METHODOLOGY}

INPUT IMAGE: Picture preparing is a strategy to play out certain procedure on a picture, to get an improved picture or to remove some helpful data from it. It is a kind of sign handling wherein input is a picture and yield might be picture or qualities/highlights related with that picture .These days, picture planning is among rapidly creating progressions. In its local shape, a design record organize picture isn't put away as a MATLAB network, or indeed fundamentally as a network. Most illustrations records start with a header containing format-specific data labels, and proceed with bitmap information that can be examined as a ceaseless stream. For this reason, you cannot utilize the standard MATLAB I/O commands stack and spare to perused and compose a design record arrange picture. Call uncommon MATLAB capacities to studied and type in picture information from illustrations record designs: To peruse a design record organize picture utilize imread.

\section{IMAGE PREPROCESSING}

Preprocessing procedures are required on colour, grey-level or parallel record pictures containing content and/or design. In character acknowledgment frameworks most of the applications utilize dark or parallel pictures since handling colour pictures is computationally tall. Such pictures may moreover contain non-uniform foundation and/or watermarks making it troublesome to extricate the record content from the picture without performing a few kind of preprocessing, subsequently; the specified result from preprocessing may be a twofold picture containing content as it were. Hence, to realize this, a few steps are required, to begin with, a few picture upgrade strategies to evacuate noise or adjust the differentiate within the picture, moment, thresholding to evacuate the foundation containing any scenes, watermarks and/or clamor, third, page division to isolated design from content, fourth, character division to partitioned characters from each other and, at long last, morphological preparing to improve the characters in cases where thresholding and/or other preprocessing strategies disintegrated parts of the characters or included pixels to them. The over methods show few of those which may be utilized in character acknowledgment frameworks and in a few applications; few or a few of these strategies or others may be utilized at diverse stages of the OCR framework. The rest of the chapter will display a few of the methods utilized amid the preprocessing organize of a character acknowledgment framework.

\section{FEATURE EXTRACTION}

Highlight extraction may be a prepare of dimensionality decrease by which an starting set of crude information is decreased to more reasonable bunches for handling. A characteristic of these expansive information sets may be a huge number of factors that require a part of computing assets to handle.

\section{- SURF- SPEEDED UP ROBUST FEATURES}

In computer vision, speeded up vigorous highlights (SURF) may be a licensed nearby highlight locator and descriptor. It can be utilized for errands such as protest acknowledgment, picture enrollment, classification, or $3 \mathrm{D}$ recreation. It is somewhat motivated by the scale-invariant include change (Sift) descriptor. It is evident that the SURF descriptors or intrigued points of an picture contribute in an unexpected way on picture recovery. On the off chance that the weights or significance levels of the descriptors are received in the coordinating separate, the picture recovery can be more exact and successful. In reality, the weights can be assessed under the TF-IDF system by considering the Beyond any doubt descriptors as visual words, which in this manner has to construct a proper codebook as well as a word implanting in a common way under the BOW technique. Be that as it may, the time taken a toll of training a codebook and a modern word inserting cannot be attempted since the preparing dataset is so tremendous in our wine label picture recovery. In arrange to overcome this difficulty; we alter the TF-IDF remove into a disentangled frame which can be computed straightforwardly from certain presented capacities [1]. 


\section{EPRA International Journal of Research and Development (IJRD)}

Volume: 6 | Issue: 1 | January 2021

- Peer Reviewed Journal

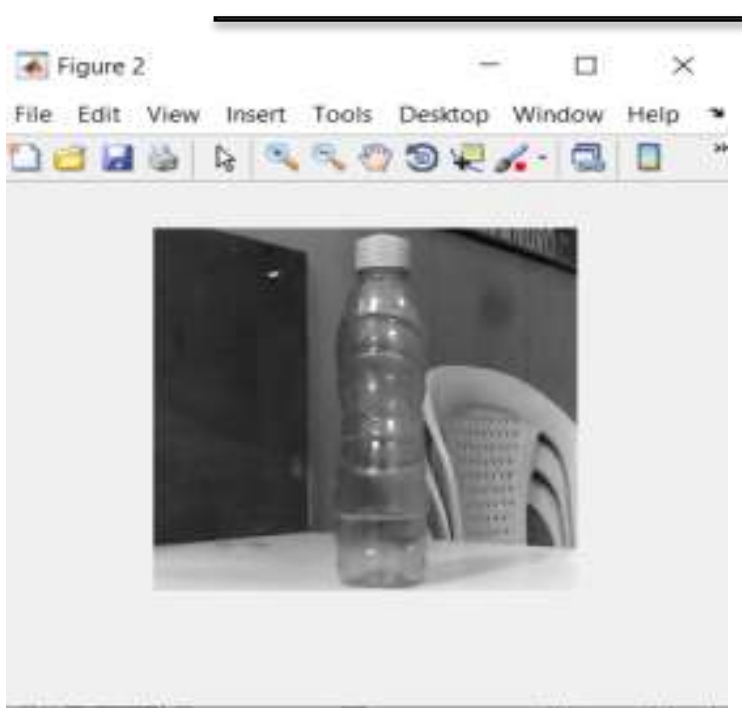

Fig: 5 Extracted images

\section{CLASSIFICATION}

Picture classification alludes to a handle in computer vision that can classify an picture concurring to its visual substance. For illustration, a picture classification calculation may be planned to tell in case an picture contains a human figure or not.

\section{- KNN CLASSIFICATION}

The k-nearest neighbours (KNN) algorithm may be a straightforward, supervised machine learning algorithm that can be utilized to solve both classification and replace issues. It's easy to execute and get it, but includes a major downside of getting to be essentially moderates as the estimate of that information in utilize grows.

A directed machine learning calculation (as contradicted to an unsupervised machine learning calculation) is one that depends on labeled input information to memorize a work that produces a suitable yield when given unused unlabeled data. A classification issue includes a discrete esteem as its output. A relapse issue contains a genuine number (a number with a decimal point) as its yield. The KNN calculation accepts that comparative things exist in near nearness. In other words, comparable things are close to each other.

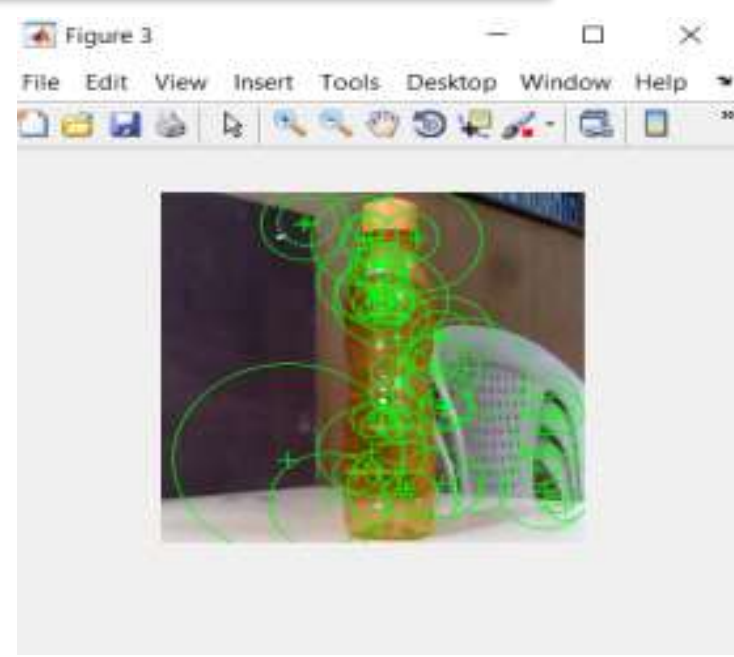

FIG: 6 KNN CLASSIFIED IMAGE

\section{IV.EXPERIMENTAL RESULT}

Amid the assessment of the plan and improvement of Keen Sensor Based Strolling Adhere it was tried in practicable and genuine time conditions. It is also evaluated by blind person. There are different conditions on different routes are chosen to check the performance. In first route, we placed dummy of wall as obstacles in numbers. The daze individual was new with this put. At first blind man used the white cane on this route and comparative performance with white can has been evaluated. The route was $27 \mathrm{~m}$ long in distance and it was covered at a speed of 0.522 $\mathrm{m} / \mathrm{s}$ with help of white cane. But when blind man used smart stick it had been covered at speed of $0.78 \mathrm{~m} / \mathrm{s}$ and with none problem. In the conclusion course appears that "Shrewd strolling adhere" has exceptional execution as compared to white cane. Likewise first route we got excellent results once we tested this stick in other routes also [2].

\section{V.CONCLUSION}

In this work is for visually impaired people to reduce the suffering from day-to-day life .This proposed idea is an attempt towards progress the life of visually reduced public by providing mobility as well as safety measures without the dependency of others. The blind people is enabled to stay connected with surrounding environment by the help of the smart stick. They would be moving safer wherever they would wish to go without getting injured from the surrounding obstacles and hurdles. This idea can be improved further by adding additional sensors thereby increasing the utility factors of the stick and the applications. 


\section{EPRA International Journal of Research and Development (IJRD) \\ Volume: 6 | Issue: 1 | January 2021 \\ - Peer Reviewed Journal}

\section{VI.FUTURE WORK}

Objects location is utilized to discover objects within the genuine world from a picture of the world that are common within the scenes of a dazzle. Based on their areas, and the camera is used to distinguish any objects. We anticipate advance changes within the future as we create unused highlight sorts counting color, separate and other features. We also suggest utilizing this component Movidius Neural Compute Adhere (NCS) could be a profound learning USB drive. The NCS is fueled by the low-power high-performance Movidius Visual Handling Unit (VPU). Run numerous gadgets on the same stage to scale execution.

\section{REFERENCE}

1. "Large Scale Category-Structured Image Retrieval for Object Identification Through Supervised Learning of CNN and SURF-Based Matching “XIAOQING LI, JIANSHENG YANG, AND JINWEN , 2020, IEEE

2. "Smart stick for blind people" N.Loganathan, K.Lakshmi,N.Chandrasekaran,S.R .Cibisakaravarthi,R.HariPriyanga,K.HarshaVart hini,2020, international conference

3. "On-the-fly Image Classification to Help Blind People”, Dalal Khalid Aljasem, Michael Heeney, Armando Pesenti Gritti, Franco Raimondi, 2016, International Conference on Intelligent Environments.

4. "New Developments in Mobility and Orientation Aids for the Blind", John a. brabyn, Member, 1982, IEEE.

5. "Wearable Obstacle Avoidance Electronic Travel Aids for Blind: A Survey”, Dimitrios Dakopoulos and Nikolaos G. Bourbakis, Fellow, 2010, IEEE.

6. “A Blind Mobility Aid Modeled After Echolocation of Bats”, Tohru Ifukube, Tadayuki Sasaki, and Chen Peng, 1991, IEEE.

7. "Obstacles Recognition System for the Blind People Using RFID”, M. Nassih, I. Cherradi, $Y$. Maghous, B. Ouriaghli and Y. Salih-Alj, 2012, IEEE.

8. "Character Detection and Recognition System for Visually Impaired People”, Akhilesh A. Panchal, Shrugal Varde, M.S. Panse, 2016, IEEE.

9. “The Navbelt-A Computerized Travel Aid for the Blind Based on Mobile Robotics Technology”, Shraga Shoval, Johann Borenstein, and Yoram Koren, 1998, IEEE.

10. "A device for human ultrasonic echolocation", Jascha Sohl-Dickstein, Santani Teng, Benjamin M. Gaub, Chris C. Rodgers, Crystal Li, Michael R. DeWeese and Nicol S. Harper, 2015, IEEE.

11. "Text-TrackingWearable Camera System for Visually-Impaired People”, Makoto Tanaka, Hideaki Goto, 2008, IEEE.
12. Elmannai W., Elleithy K. Sensor-based assistive devices for visually impaired people: Current status, challenges, and future directions. Sensors. 2017; 17:565. Doi: 10.3390/s17030565. [PMC free article] [PubMed] [CrossRef] [Google Scholar]

13. Dhod R., Singh G., Singh G., Kaur M. "Wireless Personal Communications". Volume 92. Springer; New York, NY, USA: 2017. Low cost GPS and GSM based navigational aid for visually impaired people; pp. 15751589. [Google Scholar]

14. Sylvain Cardin, Daniel Thalmann and Frederic Vexo," Wearable Obstacle Detection System for visually impaired people" Virtual Reality Laboratory (VRlab)Ecole Polytechnique Fédérale de Lausanne (EPFL)CH-1015 Lausanne, Switzerland \{sylvain.cardin, daniel.thalmann, frederic.vexo\} @epfl.ch

15. Osama Bader AL-Barrm International Journal of Latest Trends in Engineering and Technology (IJLTET)

16. B.Mohan Sitaramaiah, M.NaganaikInternational Journal of Advanced Technology in Engineering and Science www.ijates.com Volume No.03, Issue No. 01, January 2015 ISSN (online): $2348-75501 q^{\prime \prime}$.

17. F. van der Heijden, P.P.L. Regtien, "Wearable navigation assistance - a tool for the blind" MEASUREMENT SCIENCE REVIEW, Volume 5, Section 2, (2005).

18. Ankit Agarwal, Deepak Kumar, Abhishek Bhardwaj International Journal Of Engineering And Computer Science ISSN:2319-7242 Volume 4 Issue 4 (April 2015), Page No. 11375-11378

19. Srirama Divya, B.Navya, P.Suma Manasa and S.Chitra (2010). Ultrasonic and Voice Based Walking Stick for The Blind Bachelor Degree Gokaraju Rangaraju Institute Of Engineering And Technology, Hyderabad

20. Jayant Sakhardande, Pratik Pattanayak, Mita Bhowmick, -Arduino Based Mobility Canell, International Journal of Scientific \&Engineering Research, Vol. 4, Issue 4, pp 1163-1166, (April 2013). 